Bull. Korean Math. Soc. 48 (2011), No. 5, pp. 917-922

http://dx.doi.org/10.4134/BKMS.2011.48.5.917

\title{
GENERALIZED DERIVATIONS WITH ANNIHILATOR CONDITIONS IN PRIME RINGS
}

\author{
YU WANG
}

\begin{abstract}
Let $R$ be a prime ring, $H$ a generalized derivation of $R, L$ a noncentral Lie ideal of $R$, and $0 \neq a \in R$. Suppose that $a u^{s} H(u) u^{t}=0$ for all $u \in L$, where $s, t \geq 0$ are fixed integers. Then $H=0$ unless $R$ satisfies $S_{4}$, the standard identity in four variables.
\end{abstract}

Throughout this article, $R$ is always a prime ring with extended centended $C$, right Utumi quotient ring $U$, and two-sided Martindale quotient ring $Q$. The definitions and properties of these objects can be found in [3, Chapter 2]. By $S_{4}$ we denote the standard identity in four variables.

By a generalized derivation on $R$ one usually means an additive map $H$ : $R \rightarrow R$ such that $H(x y)=H(x) y+x d(y)$ for some derivation $d$ of $R$. Obviously any derivation is a generalized derivation. Another basic example of generalized derivations is the following: $H(x)=a x+x b$ for $a, b \in R$. In [12] Hvala initiated the study of generalized derivations on prime rings. In [16, Theorem 3] Lee proved the following essential result: every generalized derivation $H$ on a dense left ideal of $R$ can be uniquely extended to $U$ and assume the form $H(x)=b x+d(x)$ for some $b \in U$ and a derivation $d$ on $U$. In recent years, a number of articles discussed generalized derivations in the context of prime and semiprime rings (e.g., $[1,9,10,11,17,18,20]$ ).

In [6] Dhara and Sharma proved that, if $a \in R$ such that $a u^{s} d(u)^{n} u^{t}=0$ for all $u \in L$, a noncommutative Lie ideal of $R$, where $d$ a derivation of $R$, $s \geq 0, t \geq 0, n \geq 1$ are fixed integers, then either $a=0$ or $d=0$ unless $\operatorname{char} R=$ 2 and $R$ satisfies $S_{4}$. In [5] Dhara and Filippis proved that, if $u^{s} H(u) u^{t}=0$ for all $u \in L$, where $L$ is a noncommutative Lie ideal of $R, H$ is a generalized derivation of $R$, and $s, t \geq 0$ are fixed integers, then $H(x)=0$ for all $x \in R$ unless char $R=2$ and $R$ satisfies $S_{4}$.

Received November 18, 2009

2010 Mathematics Subject Classification. 16W25, 16N60, 16R50.

Key words and phrases. prime ring, derivation, generalized derivation, extended centroid, Utumi quotient ring.

The author has been partially supported by the Leading Academic Discipline Project of Shanghai Normal University (No.DZL803). 
In the present paper we shall extend the result of Dhara and Filippis to the situation when $a u^{s} H(u) u^{t}=0$ for all $u \in L$, where $a \in R, L$ a noncentral Lie ideal of $R, H$ a generalized derivation of $R$ and $s, t \geq 0$ are fixed integers. More precisely, our main result is the following:

Theorem 1. Let $R$ be a prime ring, $H$ a generalized derivation of $R, L$ a noncentral Lie ideal of $R$, and $0 \neq a \in R$. Suppose that $a u^{s} H(u) u^{t}=0$ for all $u \in L$, where $s, t \geq 0$ are fixed integers. Then $H=0$ unless $R$ satisfies $S_{4}$, the standard identity in four variables.

The following example illustrates the necessity of conditions in Theorem 1.

Example 1. Let $R=M_{2}(F)$, the ring of all $2 \times 2$ matrices algebra over a field $F\left(R\right.$ satisfies $\left.S_{4}\right)$. Let $H: R \rightarrow R$ such that $H(x)=e_{22} x$ for all $x \in R$. Note that $H$ is a nonzero generalized derivation of $R$. It is well-known fact that $[x, y]^{2} \in F \cdot I_{2}$ for all $x, y \in R$. Since every element in $[R, R]$ is a single commutator [2, Theorem], we see that $u^{2} \in F \cdot I_{2}$ for all $u \in[R, R]$. So, $e_{11} u^{2} H(u)=0$ for all $u \in[R, R]$.

For the proof of the main result we begin with the following simple result.

Lemma 1. Let $R$ be a prime ring with extended centroid $C$ and $a, b, c \in R$ with $a \neq 0$. If $a\left[x_{1}, x_{2}\right]^{s}\left(b\left[x_{1}, x_{2}\right]+\left[x_{1}, x_{2}\right] c\right)\left[x_{1}, x_{2}\right]^{t}=0$ for all $x_{1}, x_{2} \in R$, then either $R$ satisfies a nontrivial generalized polynomial identity (GPI) or $b+c=0$.

Proof. Suppose that $b \notin C$. We have that

$$
a\left[X_{1}, X_{2}\right]^{s}\left(b\left[X_{1}, X_{2}\right]+\left[X_{1}, X_{2}\right] c\right)\left[X_{1}, X_{2}\right]^{t}
$$

is a nonzero GPI for $R$ as it has a nonzero monomial $a\left(X_{1} X_{2}\right)^{s} b\left(X_{1} X_{2}\right)^{t+1}$. Similarly, if $c \notin C$, we also know that $R$ is a nontrivial GPI ring. Now we assume that $b, c \in C$. Then

$$
a\left[x_{1}, x_{2}\right]^{s+t+1}(b+c)=0
$$

for all $x_{1}, x_{2} \in R$. If $b+c \neq 0$, it is obvious that $a\left[X_{1}, X_{2}\right]^{s+t+1}(b+c)$ is a nonzero GPI for $R$. This proves the lemma.

The following result is crucial to the proof of our main result.

Lemma 2. Let $R=M_{m}(F)$, the ring of $m \times m$ matrices algebra over a field $F$ with $m>2$ and $0 \neq a \in R$ and $b, c \in R$ such that

$$
a\left[x_{1}, x_{2}\right]^{s}\left(b\left[x_{1}, x_{2}\right]+\left[x_{1}, x_{2}\right] c\right)\left[x_{1}, x_{2}\right]^{t}=0
$$

for all $x_{1}, x_{2} \in R$, where $s, t \geq 0$ are fixed integers. Then $b+c=0$.

Proof. Let $\varphi$ be an inner $F$-automorphism of $R$. Since

$$
a^{\varphi}\left[x_{1}, x_{2}\right]^{s}\left(b^{\varphi}\left[x_{1}, x_{2}\right]+\left[x_{1}, x_{2}\right] c^{\varphi}\right)\left[x_{1}, x_{2}\right]^{t}=0
$$

for all $x_{1}, x_{2} \in R$, we may replace $a, b$, and $c$ by $a^{\varphi}, b^{\varphi}$, and $c^{\varphi}$ respectively, to prove that $b^{\varphi}+c^{\varphi}=0$. Let $a=\sum_{i, j=1}^{m} a_{i j} e_{i j}$ where $a_{i j} \in F$. Multiplying 
$a$ by some suitable $e_{k j}$ from the left-hand side, we may assume $a=e_{k k}+$ $\sum_{s=k+1}^{m} a_{k s} e_{k s}$. Let $\varphi_{i}$ be the inner $F$-automorphism of $R$ defined by $x^{\varphi_{i}}=$ $\left(1+a_{k i} e_{k i}\right) x\left(1-a_{k i} e_{k i}\right)$ for $k+1 \leq i \leq m$. Then $a^{\varphi_{k+1}}=e_{k k}+\sum_{s=k+2}^{m} a_{k s} e_{k s}$, $a^{\varphi_{k+1} \varphi_{k+2}}=e_{k k}+\sum_{s=k+3}^{m} a_{k s} e_{k s}, \ldots, a^{\varphi_{k+1} \varphi_{k+2} \cdots \varphi_{m}}=e_{k k}$. Replacing $a, b$, $c$ by $a^{\varphi_{k+1} \varphi_{k+2} \cdots \varphi_{m}}, b^{\varphi_{k+1} \varphi_{k+2} \cdots \varphi_{m}}, c^{\varphi_{k+1} \varphi_{k+2} \cdots \varphi_{m}}$, respectively, we may write $a=e_{k k}$.

Now putting $x_{1}=e_{k i}, x_{2}=e_{i k}$ for $i \neq k$, we have

$$
\begin{aligned}
0 & =e_{k k}\left[e_{k i}, e_{i k}\right]^{s}\left(b\left[e_{k i}, e_{i k}\right]+\left[e_{k i}, e_{i k}\right] c\right)\left[e_{k i}, e_{i k}\right]^{t} \\
& =e_{k k}\left(e_{k k}+(-1)^{s} e_{i i}\right)\left(b\left(e_{k k}-e_{i i}\right)+\left(e_{k k}-e_{i i}\right) c\right)\left(e_{k k}+(-1)^{t} e_{i i}\right) \\
& =e_{k k}(b+c) e_{k k}+(-1)^{t} e_{k k}(-b+c) e_{i i}
\end{aligned}
$$

implying $(b+c)_{k k}=0$. Next putting $x_{1}=e_{k i}, x_{2}=e_{i k}+e_{i j}$ for $1 \leq i, j \leq m$ such that $k, i, j$ are mutually different, we have

$$
\begin{aligned}
0 & =e_{k k}\left[e_{k i}, e_{i k}+e_{i j}\right]^{s}\left(b\left[e_{k i}, e_{i k}+e_{i j}\right]+\left[e_{k i}, e_{i k}+e_{i j}\right] c\right)\left[e_{k i}, e_{i k}+e_{i j}\right]^{t} \\
& =\left(e_{k k}+e_{k j}\right) b\left(e_{k k}+e_{k j}+(-1)^{t+1} e_{i i}\right)+\left(e_{k k}+e_{k j}\right) c\left(e_{k k}+e_{k j}+(-1)^{t} e_{i i}\right) .
\end{aligned}
$$

Right multiplying by $e_{k k}$, we get that $\left(e_{k k}+e_{k j}\right)(b+c) e_{k k}=0$, this implies $(b+c)_{k k}+(b+c)_{j k}=0$. Since $(b+c)_{k k}=0$, we obtain that $(b+c)_{j k}=0$ for all $1 \leq j \leq m$.

Let $\psi$ be the $F$-automorphism of $R$ defined by $x^{\psi}=\left(1+e_{i k}\right) x\left(1-e_{i k}\right)$ for all $x \in R$, where $i \neq k$. Then

$$
e_{k k}^{\psi}\left[x_{1}, x_{2}\right]^{s}\left(b^{\psi}\left[x_{1}, x_{2}\right]+\left[x_{1}, x_{2}\right] c^{\psi}\right)\left[x_{1}, x_{2}\right]^{t}=0
$$

for all $x_{1}, x_{2} \in R$. Since $e_{k k}^{\psi}=e_{k k}+e_{i k}$, left multiplying by $e_{k k}$, we get

$$
e_{k k}\left[x_{1}, x_{2}\right]^{s}\left(b^{\psi}\left[x_{1}, x_{2}\right]+\left[x_{1}, x_{2}\right] c^{\psi}\right)\left[x_{1}, x_{2}\right]^{t}=0
$$

for all $x_{1}, x_{2} \in R$. As above we can obtain that $(b+c)_{j k}^{\psi}=0$ for all $j$.

On the other hand, we have

$$
\begin{aligned}
(b+c)^{\psi} & =b+c+e_{i k}(b+c)-(b+c) e_{i k}-e_{i k}(b+c) e_{i k} \\
& =b+c+\sum_{q}(b+c)_{k q} e_{i q}-\sum_{p}(b+c)_{p i} e_{p k}-(b+c)_{k i} e_{i k} .
\end{aligned}
$$

This implies that $(b+c)_{j k}^{\psi}=(b+c)_{j k}-(b+c)_{j i}$ for $j \neq i$ and

$$
(b+c)_{i k}^{\psi}=(b+c)_{i k}+(b+c)_{k k}-(b+c)_{i i}-(b+c)_{k i} .
$$

Since $(b+c)_{j k}=(b+c)_{j k}^{\psi}=0$ for all $j$, we see that $(b+c)_{j i}=0$ for all $i, j$, that is, $b+c=0$. This proves the result.

Applying the above two results we can obtain the following:

Lemma 3. Let $R$ be a prime ring with extended centroid $C$ and $a, b, c \in R$ with $a \neq 0$. If $a\left[x_{1}, x_{2}\right]^{s}\left(b\left[x_{1}, x_{2}\right]+\left[x_{1}, x_{2}\right] c\right)\left[x_{1}, x_{2}\right]^{t}=0$ for all $x_{1}, x_{2} \in R$, then $b+c=0$ unless $\operatorname{dim}_{C} R C \leq 4$. 
Proof. We assume that $\operatorname{dim}_{C} R C>4$. Our goal is to show $b+c=0$. By assumption $R$ satisfies generalized polynomial identity

$$
f\left(x_{1}, x_{2}\right)=a\left[x_{1}, x_{2}\right]^{s}\left(b\left[x_{1}, x_{2}\right]+\left[x_{1}, x_{2}\right] c\right)\left[x_{1}, x_{2}\right]^{t} .
$$

Suppose on the contrary that $b+c \neq 0$. In view of Lemma 1 we see that $R$ is a nonzero GPI ring. Since $R$ and $U$ satisfy same generalized polynomial identity (see [4]), $U$ satisfies $f\left(x_{1}, x_{2}\right)$. In case $C$ is infinite, we have $f\left(x_{1}, x_{2}\right)=0$ for all $x_{1}, x_{2} \in U \otimes_{C} \bar{C}$, where $\bar{C}$ is the algebraic closure of $C$. Since both $U$ and $U \otimes_{C} \bar{C}$ are prime and centrally closed [7], we may replace $R$ by $U$ or $U \otimes_{C} \bar{C}$ according to $C$ finite or infinite. Thus we may assume that $R$ is centrally closed over $C$ (i.e., $R C=R$ ) which is either finite or algebraically closed and $f\left(x_{1}, x_{2}\right)=0$ for all $x_{1}, x_{2} \in R$. By Martindale's theorem [19], $R$ is a primitive ring having nonzero socle $H$ with $C$ as the associated division ring. By Jacobson's density theorem [13, p. 75], $R$ is isomorphic to a dense ring of linear transformations of a vector space $V$ over $C$, and $H$ consists of the linear transformations in $R$ of finite rank.

If $\operatorname{dim}_{C} V<\infty$, then $R \cong M_{m}(C)$ for some $m>2$. By Lemma 2 we have $b+c=0$, a contradiction. Now we assume that $\operatorname{dim}_{C} V=\infty$. It is clear that there exist $h_{1}, h_{2} \in H$ such that $h_{1} a \neq 0$ and $b h_{2}+c h_{2} \neq 0$. Left multiplying $f\left(x_{1}, x_{2}\right)$ by $h_{1}$ we may assume that $a \in H$. By Litoff's theorem [8], there exists idempotent $e \in H$ such that $h_{2}, a, b h_{2}, c h_{2} \in e R e$ and $e R e \cong M_{k}(C)$ with $k>2$. Hence

$$
e a e\left[e x_{1} e, e x_{2} e\right]^{s}\left(e b e\left[e x_{1} e, e x_{2} e\right]+\left[e x_{1} e, e x_{2} e\right] e c e\right)\left[e x_{1} e, e x_{2} e\right]^{t}=0
$$

for all $x_{1}, x_{2} \in R$. Since $e a e=a \neq 0$, by Lemma 2 we have $e b e+e c e=0$. Thus

$$
b h_{2}+c h_{2}=e\left(b h_{2}\right) e+e\left(c h_{2}\right) e=e b e h_{2}+e c e h_{2}=(e b e+e c e) h_{2}=0,
$$

a contradiction.

We are ready to give:

Proof of Theorem 1. We assume that $R$ does not satisfy $S_{4}$. Our aim is to show $H=0$. By a theorem of Lanski and Montgomery [15, Theorem 13] we have $0 \neq[I, R] \subseteq L$, where $I$ is a nonzero ideal of $R$. Hence we may assume without loss of generality that $L=[I, I]$. Since $I$ and $U$ satisfy the same differential identities [4], we have

$$
a\left[x_{1}, x_{2}\right]^{s} H\left(\left[x_{1}, x_{2}\right]\right)\left[x_{1}, x_{2}\right]^{t}=0
$$

for all $x_{1}, x_{2} \in U$. By [16, Theorem 3] we may assume that $H(x)=b x+d(x)$ for all $x \in U$, where $b \in U$ and $d$ is a derivation of $U$. So

$$
a\left[x_{1}, x_{2}\right]^{s}\left(b\left[x_{1}, x_{2}\right]+d\left(\left[x_{1}, x_{2}\right]\right)\right)\left[x_{1}, x_{2}\right]^{t}=0
$$

for all $x_{1}, x_{2} \in U$. Assume first that $d$ is $Q$-inner, i.e., there exists $p \in U$ such that $d(x)=[p, x]$ for all $x \in U$. Thus

$$
a\left[x_{1}, x_{2}\right]^{s}\left((b+p)\left[x_{1}, x_{2}\right]-\left[x_{1}, x_{2}\right] p\right)\left[x_{1}, x_{2}\right]^{t}=0
$$


for all $x_{1}, x_{2} \in U$. By Lemma 3 we have $b=(b+p)-p=0$ and so

$$
a\left[x_{1}, x_{2}\right]^{s} d\left(\left[x_{1}, x_{2}\right]\right)\left[x_{1}, x_{2}\right]^{t}=0
$$

for all $x_{1}, x_{2} \in U$. It follows from [6, Theorem 1] that $d=0$ and so $H=0$ as desired.

Suppose that $d$ is not $Q$-inner. Then

$$
a\left[x_{1}, x_{2}\right]^{s}\left(b\left[x_{1}, x_{2}\right]+\left[d\left(x_{1}\right), x_{2}\right]+\left[x_{1}, d\left(x_{2}\right)\right]\right)\left[x_{1}, x_{2}\right]^{t}=0
$$

for all $x_{1}, x_{2} \in U$. In view of the powerful Kharchenko's theorem [14] we have

$$
a\left[x_{1}, x_{2}\right]^{s}\left(b\left[x_{1}, x_{2}\right]+\left[x_{3}, x_{2}\right]+\left[x_{1}, x_{4}\right]\right)\left[x_{1}, x_{2}\right]^{t}=0
$$

for all $x_{1}, x_{2}, x_{3}, x_{4} \in U$. Thus, $U$ satisfies its blended component

$$
a\left[x_{1}, x_{2}\right]^{s}\left(\left[x_{3}, x_{2}\right]+\left[x_{1}, x_{4}\right]\right)\left[x_{1}, x_{2}\right]^{t} .
$$

In particular, we have

$$
a\left[x_{1}, x_{2}\right]^{s} d\left(\left[x_{1}, x_{2}\right]\right)\left[x_{1}, x_{2}\right]^{t}=a\left[x_{1}, x_{2}\right]^{s}\left(\left[d\left(x_{1}\right), x_{2}\right]+\left[x_{1}, d\left(x_{2}\right)\right]\right)\left[x_{1}, x_{2}\right]^{t}=0
$$

for all $x_{1}, x_{2} \in U$. It follows from [6, Theorem 1] that $d=0$, a contradiction.

We conclude this paper with the following.

Conjecture. Let $R$ be a prime ring, $0 \neq a \in R, H$ a generalized derivation and $L$ a noncentral Lie ideal of $R$. Suppose that $a u^{s} H(u)^{n} u^{t}=0$ for all $u \in L$, where $s, t \geq 0$ and $n \geq 1$ are fixed integers. Then $H=0$ unless $R$ satisfies $S_{4}$, the standard identity in four variables.

\section{References}

[1] E. Albas, N. Argac, and V. D. Fillippis, Generalized derivations with Engel conditions on one-sided ideals, Comm. Algebra 36 (2008), no. 6, 2063-2071.

[2] A. A. Albert and B. Muckenhoupt, On matrices of trace zero, Michigan Math. J. 4 (1957), 1-3.

[3] K. I. Beidar, W. S. Martindale III, and A. V. Mikhalev, Rings with Generalized Identities, Marcel Dekker, New York-Basel-Hong Kong, 1996.

[4] C. L. Chuang, GPIs having coefficients in Utumi quotient rings, Proc. Amer. Math. Soc. 103 (1988), no 3, 723-728.

[5] B. Dhara and V. De Filippis, Notes on generalized derivations on Lie ideals in prime rings, Bull. Korean Math. Soc. 46 (2009), no. 3, 599-605.

[6] B. Dhara and R. K. Sharma, Derivations with annihilator conditions in prime rings, Publ. Math. Debrecen 71 (2007), no. 1-2, 11-20.

[7] T. S. Erickson, W. S. Martindale III, and J. M. Osborn, Prime nonassociative algebras, Pacific J. Math. 60 (1975), no. 1, 49-63.

[8] C. Faith and Y. Utumi, On a new proof of Litoff's theorem, Acta Math. Hungar 14 (1963), 369-371.

[9] V. De Filippis, An Engel condition with generalized derivations on multilinear polynomials, Israel J. Math. 162 (2007), 93-108.

[10] _ Posner's second theorem and an annihilator condition with generalized derivations, Turkish J. Math. 32 (2008), no. 2, 197-211.

[11] Generalized derivations in prime rings and noncommutative Banach algebras, Bull. Korean Math. Soc. 45 (2008), no. 4, 621-629. 
[12] B. Hvala, Generalized derivations in rings, Comm. Algebra 26 (1998), no. 4, 1147-1166.

[13] N. Jacobson, Structure of Rings, Amer. Math. Soc. Colloq. Pub., 37, Amer. Math. Soc., Providence, RI, 1964.

[14] V. K. Kharchenko, Differential identities of prime rings, Algebra Logika 17 (1978), no. 2, 220-238, 242-243.

[15] C. Lanski and S. Montgomery, Lie structure of prime rings of characteristic 2, Pacific J. Math. 42 (1972), 117-136.

[16] T. K. Lee, Generalized derivations of left faithful rings, Comm. Algebra 27 (1999), no. 8, 4057-4073.

[17] T. K. Lee and W. K. Shiue, Identities with generalized derivations, Comm. Algebra 29 (2001), no. 10, 4437-4450.

[18] J. S. Lin and C. K. Liu, Generalized derivations with invertible or nilpotent on multilinear polynomials values, Comm. Algebra 34 (2006), no. 2, 633-640.

[19] W. S. Martindale III, Prime rings satisfying a generalized polynomial identity, J. Algebra 12 (1969), 576-584.

[20] Y. Wang, Generalized derivations with power-central values on multilinear polynomials, Algebra Colloq. 13 (2006), no. 3, 405-410.

Mathematics and Science College

Shanghai Normal University

Shanghai 200234, P. R. China

E-mail address: ywang2004@126.com 